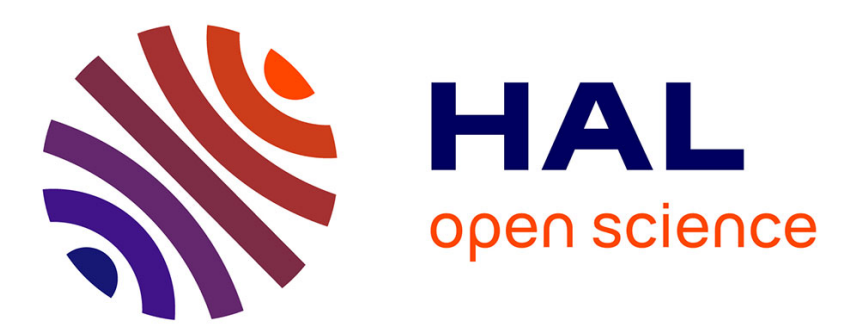

\title{
Une machine à retraiter les outils de mesure du crime et de l'insécurité: l'Observatoire National de la Délinquance
}

Frédéric Ocqueteau

\section{To cite this version:}

Frédéric Ocqueteau. Une machine à retraiter les outils de mesure du crime et de l'insécurité: l'Observatoire National de la Délinquance. Droit \& Société, 2012, 81, pp.447-471. hal-00744408

\section{HAL Id: hal-00744408 \\ https://hal.science/hal-00744408}

Submitted on 23 Oct 2012

HAL is a multi-disciplinary open access archive for the deposit and dissemination of scientific research documents, whether they are published or not. The documents may come from teaching and research institutions in France or abroad, or from public or private research centers.
L'archive ouverte pluridisciplinaire HAL, est destinée au dépôt et à la diffusion de documents scientifiques de niveau recherche, publiés ou non, émanant des établissements d'enseignement et de recherche français ou étrangers, des laboratoires publics ou privés. 


\title{
Une machine à retraiter les outils de mesure du crime et de l'insécurité : l'Observatoire national de la délinquance
}

\author{
Frédéric Ocqueteau
}

Centre de Recherches Sociologiques sur le Droit et les Institutions Pénales (CESDIP), Immeuble Edison, 43 boulevard Vauban, F-78280 Guyancourt.

<ocqueteau@cesdip.fr>

Résumé

L'article retrace le travail quotidien de l'Observatoire national de la délinquance (OND), dispositif institué en 2004 par le ministère de l'Intérieur français pour rassembler et diffuser des sources de connaissances dispersées et fragmentaires sur l'évolution statistique des crimes et délits et mieux cibler l'action publique préventive et répressive. Il explique les contraintes socio-techniques des opérations de rapprochement des données de la police et de la gendarmerie, et insiste sur les réticences du ministère de la Justice. Il montre comment la mobilisation d'enquêtes nationales de victimes conçues comme des données alternatives aux outils bureaucratiques traditionnels perturbe le processus général d'harmonisation et d'intégration. Il éclaire les méandres de la mise en œuvre inachevée d'une "activité administrative constituante » dans un contexte ultra politisé.

Action publique - Bureaucraties policières - Criminalités - Instruments de mesure-Observatoire.

\section{Summary The OND: A Machine to Reprocess Tools Measuring Crime and Insecurity}

This paper recounts the daily work of the National Observatory of Delinquency (OND), which was established in 2004 by the French Ministry of the Interior both to collect and distribute data, often scattered and fragmentary, on the statistical evolution of crimes and offenses, and to improve crime prevention and crime-fighting measures. It explains the socio-technical constraints of bringing together policing data by the national police and gendarmerie, and it insists on the Ministry of Justice's reticence. This article shows how mobilizing national victim surveys, conceived as alternative data to the traditional bureaucratic tools, disrupts the general process of harmonization and integration. It illuminates the meanders of the unfinished implementation of a "constituent administrative activity" in a highly politicized context.

Crime - Measuring instruments - Policing bureaucracies - Public policy Observatory. 
Dans une conjoncture où la nécessité de mieux répondre à la demande de protection des citoyens est devenue une obsession électorale et un enjeu ultra politisé, il était inévitable que les performances des administrations régaliennes de l'État, police et justice, fassent l'objet auprès de la Nation d'une "redevabilité » ${ }^{1}$ plus transparente. Les demandes adressées aux détenteurs et interprètes des statistiques des délinquances et des risques, au cours de la dernière décennie, ont durablement fixé l'attention française sur certaines dimensions controversées des politiques de sécurité : celles qui prétendent prouver l'efficacité des politiques de fermeté dites de « tolérance zéro » 2 importées des États-Unis par le biais d'une publicité périodique de chiffres commentés en direction des journalistes intéressés à établir des liens entre demandes citoyennes et offres étatiques. À ce sujet, la reddition des comptes de l'État s'est progressivement concentrée dans les mains du seul ministère de l'Intérieur, ministère qui, sous la dernière décennie considérée, s'est annexé l'interprétation de l'évolution de tous les phénomènes illégaux, au détriment du ministère de la Justice ou d'autres ministères ${ }^{3}$.

Le vieux Compte général annuel de la Justice de $1828^{4}$, qui avait servi durant cent cinquante ans de seul thermomètre pour mesurer l'évolution statistique du crime, fut quasiment abandonné dans les années 1980. Il ne parvenait plus à rendre compte avec finesse de l'évolution des crimes et délits, alors que d'autres outils de mesure aux mains des agences policières passaient, aux yeux de l'État, pour beaucoup plus fiables que ceux de l'administration judiciaire.

$\mathrm{Au}$ demeurant, une bonne connaissance scientifique de la construction de la " chaîne pénale " par le biais de l'image inversée de l'entonnoir à filtres successifs 5 avait permis d'identifier les raisons pour lesquelles les statistiques policières reflétaient bien plus finement les évolutions de la criminalité apparente ${ }^{6}$ que ne le faisaient les outils de mesure de la Justice. Les comptes de la Justice pénale restaient largement conditionnés et surdéterminés par les activités proactives et réactives de la police ${ }^{7}$, s'agissant des résultats du produit du travail des tribunaux répressifs et de l'administration pénitentiaire. Beaucoup d'analystes expliquèrent le «tournant

1. Traduction approximative de l'excellent syntagme britannique d'accountability.

2. Jacques DE MAILLARD et Tanguy LE GOFF, « La tolérance zéro en France. Succès d'un slogan, illusion d'un transfert ", Revue française de science politique, 59 (4), 2009, p. 655-679.

3. Luc RudolPH et Christophe Soullez, Les stratégies de la sécurité 2002-2007, Paris : PUF, 2007. Dans cet ouvrage de circonstance paru à la veille des élections présidentielles, co-écrit par le directeur de la cellule statistique de l'Observatoire national de la délinquance, on peut lire ceci : "Tant qu'il n'y aura pas de politique transversale de lutte contre l'insécurité [...], que n'auront pas été listés tous les sujets relatifs à ce thème à travers tous les ministères, et tant qu'ils n'auront pas été mis "sous tutelle" du ministère de l'Intérieur, la lutte contre l'insécurité restera une utopie» (p. 212).

4. Michèle PERROT et Philippe RoBert, Compte général de l'administration de la Justice criminelle en France pendant l'année 1880 et rapport relatif aux années 1826 à 1880, Genève : Slatkine reprints, 1989.

5. Bruno AuBuSSON DE CAVARLAY, «De la statistique criminelle apparente à la statistique judiciaire cachée ", Déviance et société, 22 (2), 1998, p. 155-180.

6. Cécile BARBERger-DAmAmme, De la criminalité apparente. Théorie et pratique à partir de trois années de rapports journaliers des polices urbaines du Rhône, thèse de doctorat, Lyon : Université Lyon 3, 1981.

7. René LÉVY, Du suspect au coupable : le travail de police judiciaire, Genève : Médecine et Hygiène, Paris : Méridiens Klincksieck, coll. «Déviance et société », 1987. 
répressif » affectant l'ensemble de nos démocraties ${ }^{8}$ comme une forme d'adaptation à une fragilisation générale liée à la globalisation de l'économie de marché et à l'entrée dans "l'ère des risques " 9 : le recul des États-providence 10 et la punitivité corrélative des État néolibéraux 11 expliquaient une nouvelle propension à la contention de populations précaires, d'autant plus aisément criminalisées que diminuaient les filets de protection traditionnels des États-providence. Enfin, la montée de l'idéo logie du management des services publics et du besoin d'en mieux mesurer le rendement par une approche coût/efficacité issue des référentiels de l'économie marchande était expliquée par la pression internationale à la compétition des États et à leur entrée dans les normes plus contraignantes du benchmarking ${ }^{12}$.

Personne n'est pour l'instant en capacité d'imposer une explication unifiée des liens possibles entre des phénomènes de cette envergure parce que les lectures des effets suscités par le «nouvel esprit du capitalisme" sont encore loin d'être consensuelles. Reste que, dans ce contexte général, le pilotage des politiques de sûreté/ sécurité pose des questions assez sensiblement différentes de celles du passé. Les controverses publiques liées aux instruments de mesure des «insécurités », des « situations criminogènes », des « situations à risques » ou des « comportements probléma tiques » contemporains occupent à cet égard une place déterminante. Ce qui était moins le cas naguère, d'une part, lorsque les indicateurs de mesure de ces phénomènes arrivaient en ordre dispersé dans la connaissance fragmentaire qu'en avaient les différents ministères et, d'autre part, quand les objectifs de sûreté (pronostics) restaient peu interrogés à partir des thermomètres (diagnostics) sur les résultats desquels ils étaient censés se fonder. Les amalgames et confusions politico-partisanes pouvaient alors indéfiniment prospérer et déboucher sur les conclusions les plus binaires lors des échéances électorales : puisque personne ne s'entendait sur les fièvres signalées par les divers thermomètres de l'État, il suffisait de s'en prendre aux instruments et de les casser (théories radicales), ou de proposer d'en construire de nouveaux (théories réformistes). Ce qui faisait assez peu avancer la connaissance du travail concret de l'État en ce domaine.

Les gestes déontologiques radicaux 13 signalent pourtant souvent quelque chose de précieux au sujet de la culture politique française : les outils de mesure des problèmes sociaux et/ou de gestion des performances des services publics pour les combattre ou les solutionner sont des outils ultra sensibles, à manier avec d'infinies précautions. Deux démonstrations récentes en ont été fournies par les débats très

8. Nicolas CARRIER, «Sociologies du virage punitif», Champ Pénal/Penal Field, 7, $2010<$ http://champpenal. revues.org/7818>.

9. Ulrich BECK, Risikogesellschaft, Francfort : Surkhamp, 1986 (en français : La société du risque. Sur la voie d'une autre modernité, traduit de l'allemand par Laure Bernardi, Paris : Aubier, 2001).

10. Voir d'importantes nuances de ce lien dans la comparaison internationale établie par Hugues LAGRANGE, Demandes de sécurité, France, Europe, États-Unis, Paris : Seuil, 2003.

11. Loïc WACQUANT, Punir les pauvres, le nouveau gouvernement de l'insécurité sociale, Marseille: Agone, 2004 ; Robert CASTEL, L'insécurité sociale, qu'est-ce qu'être protégé ?, Paris : Seuil, 2003.

12. Luc Boltanski et Ève CHIAPELlo, Le nouvel esprit du capitalisme, Paris : Gallimard, 1999.

13. Association PÉnOmbre, Chiffres en folie. Petit abécédaire de l'usage des nombres dans le débat public et les médias, Paris : La Découverte, 1999. 
vifs liés à la pertinence de la mobilisation des statistiques dites « ethniques » 14 pour mieux cibler des populations discriminées, ou au contenu de certains fichiers policiers ${ }^{15}$ pour rendre plus efficaces les services dans la lutte contre le récidivisme.

Quels que soient les arguments qui s'affrontent au sujet de l'utilité des outils de mesure et des objectifs qu'ils servent, ils se présentent fréquemment comme des acteurs actifs des politiques publiques, y compris dans le domaine de l'offre de sûreté. Dans le droit fil de travaux pionniers en sciences politiques ${ }^{16}$, nous sommes fondés à évoquer des acteurs actifs, dans la mesure où ces instruments de connaissance sont devenus les premiers "pilotes» de la mise en œuvre des injonctions politiques édictées par les gouvernants. Témoin, le fameux Compstat importé des États-Unis, qui, largement mobilisé à la Préfecture de police ${ }^{17}$, fait désormais partie d'un langage managérial fédérateur au sein de tous les services publics de police occidentaux pour optimiser le coût par rapport à l'efficacité des solutions aux problèmes de sûreté.

La métaphore des instruments de mesure comme pilotes possibles de l'action publique s'inscrit dans un puissant courant de pensée d'une sociologie politique nourrie de travaux sur l'action organisée ou l'action stratégique 18. Beaucoup d'entre eux portent moins sur la substance discursive ou intentionnelle des outils mobilisés par les managers ou pilotes de l'action publique, que sur leurs instrumentations techniques, scientifiques ou gestionnaires pour une meilleure conduite de l'action collective et promotion de nouvelles capacités stratégiques. Maintes approches postulent l'efficacité des outils, en les considérant comme un moyen simple de prouver comment une équipe dirigeante parvient aux fins recherchées. Cette conception prend généralement place dans la vision classique des modes de gouvernance d'administration publique restant structurés par un certain nombre de contraintes spécifiques, dont la principale d'entre elles serait que les finalités politiques restent largement extérieures aux bureaucraties d'exécution ${ }^{19}$. Les vi-

14. Collectif CARSED, Le retour de la race, contre les "statistiques ethniques", La Tour d'Aigues: L'Aube, 2009.

15. COMmission NATIONALE DE L'INFORMATIQUE ET DES LIBERTÉS (CNIL), «Contrôle du STIC: les propositions de la CNIL pour une utilisation du fichier plus respectueuse du droit des personnes", 21 janvier 2009, $<$ http://www.cnil.fr/la-cnil/actu-cnil/article/browse/3/article/505/controle-du-stic-les-propositions-de-la-cnilpour-une-utilisation-du-fichier-plus-respectueuse-du/>.

16. Pierre Lascoumes et Patrick Le GalÈs (dir.), Gouverner par les instruments, Paris : Presses de Sciences Po, 2004.

17. David Dufresne, « Médiapart révèle le tableau de bord stratégique de la préfecture de police de Paris », Mediapart, 28 mai 2008 <http://www.mediapart.fr/journal/france/280508/mediapart-revele-le-tableau-debord-strategique-de-la-prefecture-de-police-de-\#comment-181393> ; Emmanuel DIDIER, " "Compstat" à Paris : initiative et mise en responsabilité policière », Champ pénal/Penal Field, VIII, 2011 <http://champ penal.revues.org/7971>.

18. Christopher Hood, The Tools of Government, Chatham (NJ) : Chatham House, 1986; Christopher HooD, "Contemporary Public Management: A New Paradigm?», Public Policy and Administration, 10 (2), 1995, p. 104-117 ; Pierre LASCOUMES et Patrick LE GALÈS, «L'action publique saisie par ses instruments ", in Pierre LASCOUMES et Patrick LE GALÈs (dir.), Gouverner par les instruments, op. cit., p. 11-44 ; Alain DeSROSIÈRES, Pour une sociologie historique de la quantification. L'argument statistique I, Paris :Presses de l'École des Mines, 2008 ; Alain DeSROSIÈRES, Gouverner par les nombres. L'argument statistique II, Paris : Presses de l'École des Mines, 2008.

19. James Q. Wilson, Bureaucracy, New York: Basic Books, 1989. 
sions médiatrices des outils de gestion publique qui en résultent ont été bien mises en évidence par les travaux de Jean-Claude Moisdon. Ce chercheur avait défini les outils comme une «formalisation de l'activité organisée, de ce qu'elle est ou de ce qu'elle sera (ensemble de raisonnements et de connaissances pour instruire les actes de la trilogie : prévoir, décider, contrôler) " 20 . Quelques années plus tard, il précisa sa pensée dans le sillon de l'école de l'économie des conventions, en les considérant comme « une abstraction, un modèle, petit ou gros, qui relie entre elles plusieurs quantités (des productions, des prix, des nombres de défauts, des effectifs de personnel, etc.) »21. Son apport intellectuel s'est avéré décisif pour distinguer les outils de gestion des règles (prescriptives) ou des dispositifs les guidant vers des finalités assignées. À sa suite, l'on s'est avisé que les instruments de mesure pouvaient également participer à la transformation des activités et des organisations elles-mêmes. Au sein des organisations de la sécurité publique, nous avons montré, par exemple, comment la mise à plat de l'ensemble des tableaux de bord disponibles dans tout commissariat de police français pour agir sur l'environnement et mieux gérer les ressources humaines permettait aux échelons supérieurs de l'organisation de faire entrer dans les faits les discours de performance et de rentabilité des services de police sous leur contrôle ${ }^{22}$.

Or, depuis quelques années, un "observatoire chapeau " fortement médiatisé est venu s'immiscer dans l'usage traditionnel des outils de mesure des directions centrales et départementales de la sécurité publique française. Nous avons montré à ce sujet comment l'Observatoire national de la délinquance (OND) avait commencé à acquérir une certaine influence dans son mandat d'inventorier et de recueillir différentes séries de données sur les phénomènes délinquants 23 . Nous avions alors replacé sa genèse et son apparition au sein d'une expansion générale d'observatoires de toutes natures conçus comme des espaces stratégiques de dialogues 24 , à la faveur notamment d'une diminution perceptible de l'emprise de l'intégrisme scientifique dans le domaine de l'interprétation quantifiée du crime 25

20. Jean-Claude Moisdon, Du mode d'existence des outils de gestion. Les instruments de gestion à l'épreuve de l'organisation, Paris : Seli Arslan, 1997, p. 7.

21. Jean-Claude MoISDON, "Comment apprend-on par les outils de gestion? Retour sur une doctrine d'usage ", in Régine TEULIER et Philippe LORINO (dir.), Entre connaissance et organisation : l'activité collective, Paris : La Découverte, 2005, p. 239-250.

22. Frédéric OCQUETEAU, La sécurité publique à l'épreuve de la LOLF, Paris : CERSA-CNRS, 2008 <http://halshs. archives-ouvertes.fr/docs/00/41/84/73/PDF/La_securite_publique_a_l_epreuve_de_la_LOLF.pd\$; ID., "Qu'est-ce qu'un service de sécurité publique rentable? ", in OBSERVATOIRE NATIONAL DE LA DÉLINQUANCE (OND), $L a$ criminalité en France. Rapport 2008, Paris : CNRS éditions, 2008, p. 337-358. Voir aussi Anaïk PURENNE et Jérôme AUST, «Piloter la police par les indicateurs? Effets et limites des instruments de mesure des performances ", Déviance et société, 34 (1), 2010, p. 7-28.

23. Frédéric Ocqueteau, "Observer les délinquances. Où, comment et pourquoi ? Sur la genèse de l'Observatoire national de la délinquance", in Jérôme FerRet et Christian MouHANnA (dir.), Peurs sur la ville, vers un populisme punitifà la française, Paris : PUF, 2005, p. 188-210.

24. Pierre LASCOUMES et Jean-Pierre LE BOURHIS, Administrer les pollutions et les nuisances, Paris : L'Harmattan, 1997.

25. On évoque par là cette propension, toute franco-française, à croire que l'interprétation critique légitime des données quantifiées sur les phénomènes criminels ne devrait appartenir qu'aux « scientifiques ", comme si une barrière infranchissable les opposait à d'autres interprètes moins légitimes. Cette croyance est fondée sur le besoin d'une mise à distance des premiers à l'égard des seconds, réputés moins outillés 
et du besoin ressenti de renouveler les différentes approches, par le biais d'une meilleure connaissance de la construction de tous les outils disponibles.

Mais, faute de distance suffisante, l'inventaire plus précis du rôle singulier joué par cet organisme dans la structuration de la rentabilité de la production de la sécurité publique en France restait à établir. C'est pourquoi, face au besoin de multiplier les monographies dans les lieux institutionnels mêmes où, empiriquement, se concentre la capitalisation des données, s'élabore la digestion et se formatent certains savoirs quantifiés pour l'État, nous proposons de montrer comment cet observatoire 26 confirme l'hypothèse d'école d'une activité administrative constituante. Suivant en cela Philippe Bezès et Odile Join-Lambert 27 , on entend "des activités administratives » mettant en place des cloisons pour les administrations publiques, orientant fortement les déplacements entre elles selon des degrés de contrainte variables, tout en les façonnant par l'organisation de leurs fonctions structurantes, dans leurs attributions d'enregistrement, de comptage, d'allocation et d'expertise. La démonstration d'ensemble sera effectuée en trois étapes.

\section{Emmagasiner et rationaliser des données administratives disparates}

Avant de montrer les mécanismes empiriques des contributions de l'OND comme réceptacle et retraiteur des informations collectées sur les délinquances, il convient de retracer succinctement les étapes clés de l'institutionnalisation de ce dispositif durant la dernière décennie. En 2001, à l'approche des élections présidentielles, le Premier ministre Lionel Jospin, qui a fait acte de candidature, se montre alarmé par les rapports de ses services sur les statistiques dégradées des crimes et délits de l'année 2000. En juillet 2001, le sénateur de droite Robert Pandraud, ancien ministre de la Sécurité de la première cohabitation politique (19861988), et le député de gauche Christophe Caresche sont chargés de l'élaboration d'un rapport sur la mise en place de nouveaux instruments statistiques. «Ces instruments, précisait la feuille de route, doivent être aptes à rendre compte de l'évolution réelle de la délinquance, de l'activité des services d'enquête comme des suites données par l'institution judiciaire, permettre de passer d'une logique de constatation des infractions à une logique de mesure de l'insécurité et des résultats

épistémologiquement, mais, hélas pour eux, beaucoup plus écoutés par les politiques et les médias que naguère. Il s'ensuit une tension très vive entre les sphères d'expertise, où les premiers sont en passe de perdre virtuellement la main, du haut d'une morale de conviction prêchant un peu dans le désert.

26. Le matériau de l'article est constitué par une observation de cinq années au sein du conseil d'orientation de l'OND en tant que "membre du monde scientifique ou universitaire " (2004-2009), d'après la terminologie retenue; la tenue d'un journal des séances auxquelles nous avons assisté (7 par an); une participation régulière au sein d'un sous-groupe de techniciens des statistiques de faits d'insécurité des transports publics durant deux ans (2005-2006); des entretiens et correspondances privées avec les membres du conseil d'orientation et de la "cellule statistique», en évaluation de ses propres travaux avant leurs publications; des contributions personnelles pré-négociées dans les supports de l'organisme, et des prises de position officielles et officieuses, dissidentes ou non, au sujet des orientations arrêtées.

27. Philippe Bezès et Odile JoIn-LAmberT, «Comment se font les administrations : analyser des activités administratives constituantes ", Sociologie du travail, 52 (2), 2010, p. 133-150, spécialement p. 143. 
obtenus ${ }^{28}$. " Le rapport des deux parlementaires est déposé en janvier 2002. Il inventorie l'état des divers instruments de mesure utilisés pour chiffrer les délinquances, les réponses données, et leur consistance. Il montre l'absence de coordination et l'éparpillement des sources, le cloisonnement des systèmes d'information entre ministères et l'impossibilité de croiser les nomenclatures, et conclut à une vision partielle voire faussée des délinquances à l'échelon national. Les auteurs suggèrent alors de mettre en place une structure autonome et indépendante qui recueillerait l'ensemble des infractions recensées par les diverses administrations, sans porter atteinte aux outils de lecture traditionnels dont sont dotés les services du ministère de l'Intérieur. Ils plaident pour une nouvelle structure qui aiderait à mieux piloter les forces de prévention et de répression sur les délinquances les plus problématiques.

Le nouveau ministre de l'Intérieur du président Chirac réélu, Nicolas Sarkozy, reprend l'idée de création d'un observatoire dont la mise en place du projet n'avait pu aboutir à cause des échéances électorales, et décide sans plus attendre que les statistiques seront désormais publiées en ligne mensuellement. En novembre 2003, un OND est installé dans les nouveaux locaux de l'Institut national des hautes études de sécurité (INHES) à la Plaine Saint-Denis, se présentant d'emblée comme un outil retirant partiellement la production des statistiques de délinquance (construction et commentaire) du "domaine réservé » du ministre de l'Intérieur. Il est pensé comme étant l'un des deux départements du nouvel Institut des hautes études de sécurité (ex-IHESI), établissement public administratif, avec le département de la Formation et des Études. Sa logistique est placée sous l'autorité du directeur de l'INHES, assisté d'un chef de département. "L'indépendance stratégique» de l'organisme est en théorie garantie par son conseil d'orientation qui «décide et détermine les objectifs des études et recherches dans les domaines propres de l'OND, et pren[d] en compte les demandes d'études exprimées par le ministère de l'Intérieur ", formulation assez alambiquée qui ne manquera pas de susciter par la suite bien des commentaires sur l'étendue de son exact mandat. Présidé par le directeur d'un cabinet de conseil en sécurité, « expert » autoproclamé, très influent auprès de certains cercles policiers et militaires, le conseil d'orientation du nouvel OND a en effet pour vocation de définir des stratégies garantissant la fiabilité et la pertinence des données statistiques en matière de sécurité 29.

28. Christophe CARESCHE et Robert PANDRAUD, Rapport sur la création d'un observatoire de la délinquance, Paris : La Documentation française, 2003 ; citant les lettres 872 et 873/01CG du Premier ministre en date du 23/07/2001.

29. Notre entrée y a été facilitée par la publication, une année plus tôt, d'un ouvrage parallèle faisant un état des lieux sur les indicateurs du crime : Frédéric OCQUETEAU, Jacques FrenAIS et Pierre VARLY, Ordonner le désordre, une contribution au débat sur les indicateurs du crime, Paris: La Documentation française, 2002, et par l'invitation pressante de nous voir contribuer aux discussions internes de l'organisme, y compris de rendre publiques nos opinions quand elles divergeaient des décisions arrêtées. Une attitude dont seul, pour sa part, le sénateur PS Jacques Mahéas fit un large usage durant tout son mandat. Pour faciliter la lisibilité ultérieure, nous citerons les rapports annuels de l'OND, publiés sous la direction de l'INHES à La Documentation française pour les deux premières années, ou sous celle d'Alain Bauer aux éditions du CNRS pour les trois suivantes: OND, Rapport 2005 ; OND, Rapport 2006 ; OND, Rapport 2007 ; OND, Rapport 2008; OND, Rapport 2009. Plus généralement, sur la position de sociologues invités dans les organismes publics, voir le témoignage de Dominique SCHNAPPER, Une sociologue au Conseil constitutionnel, 
Les premiers travaux de l'Observatoire portent sur l'élaboration d'une méthode d'analyse et d'interprétation des statistiques sur les faits de crimes et délits constatés et élucidés par les services de police et les unités de gendarmerie. Une réflexion concertée du conseil d'orientation avec la DGPN 30 et la DGGN ${ }^{31}$ conduit à la décision de dissocier la lecture des 107 catégories de crimes et délits récurrents nomenclaturés, à partir du principal fichier policier d'alimentation, le STIC ${ }^{32}$, en quatre rubriques agrégées de manière plus cohérente et lisible : " atteintes volontaires à l'intégrité physique "; "atteintes aux biens"; " escroqueries et infractions économiques et financières ", et « infractions révélées par l'activité des services » (IRAS) 33 . Cette suggestion en a été assez facilement actée. Notons que cette première rationalisation concrète des agrégats de transgressions aux incriminations pénales par une répartition différenciée des crimes et des délits selon les deux grandes modalités de pratiques policières réactives et proactives ne s'était encore jamais traduite aussi clairement dans les documents ministériels ${ }^{34}$. Le nouveau mode de lecture des statistiques proposé met un terme explicite à l'objection classique en France, reprise des criminologues américains John Kitsuse et Aaron Cicourel ${ }^{35}$, sur le sens qu'il convient d'attribuer à la construction des statistiques policières. Ceux-ci avaient en effet estimé que les

Paris : Gallimard, 2010. Plus spécifiquement, sur la position de chercheurs du CNRS mis à disposition du ministère de l'Intérieur, à l'IHESI notamment, voir Frédéric OCQUETEAU et Dominique MONJARDET, «Insupportable et indispensable, la recherche au ministère de l'Intérieur », in Philippe BEZÈs, Michel CHAUvIÈRE, Jacques CHevallier, Nicole DE Montricher et Frédéric OCQUeteau (dir.), L'État à l'épreuve des sciences sociales. La fonction recherche dans les administrations sous la V République, Paris : La Découverte, 2005, p. 229-247. Sur la posture d'un sociologue conseiller à la DGPN, voir Dominique MONJARDET, «L'information, l'urgence et la réforme. Réflexions sur le fonctionnement de la direction centrale de la Sécurité publique ", in Sebastian RocHÉ (dir.), Réformer la police et la sécurité. Les nouvelles tendances en France et aux État-Unis, Paris : Odile Jacob, 2004, p. 128-142. Ou, au Québec, à titre de comparaison : Maurice CHALOM, «Descente chez les bleus : une expérience professionnelle au sein de la police montréalaise ", Revue française d'administration publique, 118, 2006, p. 281-290.

30. Direction générale de la police nationale.

31. Direction générale de la gendarmerie nationale.

32. Le STIC (système de traitement des infractions constatées), également connu sous le nom familier d' ' index 4001 ", est un fichier de police informatisé regroupant les informations relatives aux circonstances de temps et de lieu de la commission des infractions constatées ou élucidées par les services de police nationale dans leurs activités de police judiciaire. Il comprend des données relatives aux personnes théoriquement mises en cause au cours de l'enquête de police ainsi qu'aux victimes de ces infractions. Existant informellement depuis 1971, il n'a été légalisé que vingt ans plus tard, en 2001 (décret du 5/07/2001). Il est placé sous la responsabilité juridique du directeur général de la Police nationale, et est géré par la sousdirection de la police technique et scientifique de la direction centrale de la Police judiciaire. Deux de ses finalités principales furent précisées par l'article 21 de la loi du 18 mars 2003 : (1) «faciliter la constatation des infractions à la loi pénale, rassembler des preuves de ces infractions et rechercher leurs auteurs "; (2) «exploiter des informations recueillies à des fins statistiques». Au regard de ses usages policiers réels souvent abusifs, ce fichier est aujourd'hui soumis à de nombreuses contestations, et fait même l'objet d'une interrogation sur sa légalité constitutionnelle. Sur tous ces points, voir Philippe PICHON et Frédéric OCQUETEAU, Une mémoire policière sale : le fichier STIC. Témoignages croisés d'un policier et d'un sociologue, Paris : Jean-Claude Gawsewitch, 2010.

33. Concrètement, tout ce qui ressort de l'activité proactive de la police, et notamment cette délinquance de masse dite sans victime, parmi laquelle les contentieux d'ILE (infractions à la législation sur les étrangers) et d'ILS (infractions à la législation sur les stupéfiants).

34. OND, Rapport 2005.

35. John I. Kitsuse et Aaron V. Cicourel, «A Note on the Uses of Official Statistics », Social Problems, 11 (2), 1963, p. 131-139. 
indicateurs administratifs des crimes et délits devaient être lus, dans le débat public, non point comme les reflets d'une réalité sui generis des "délinquances" commises, mais comme une construction basée sur les investissements bureaucratiques de la police elle-même. Il fallut pas moins de quarante années et des centaines de débats savants pour que cette évidence finisse par s'imposer partiellement à l'entendement des hauts fonctionnaires de l'appareil de l'État, autrement dit pour qu'en soit actée une certaine forme de traduction opérationnelle. Or, cette possibilité ne survint que parce que les «nouveaux agrégats » proposés par l'OND ne remettaient pas en cause les fondements sur lesquels reposaient les séries statistiques issues du fichier STIC, sa source d'alimentation principale. L'OND délaissait volontairement tout le champ problématique des infractions contraventionnelles, faute de pouvoir d'emblée traiter simultanément toute la matière disponible, ce qui devait susciter de nombreuses critiques au sujet des effets pervers engendrés dans les modalités d'enregistrement des faits 36 .

Pour faire admettre cette audace aux directions centrales, le conseil d'orientation de l'OND adopta d'abord, à l'égard de l'enjeu de son alimentation à la base dont il n'avait pas statutairement à connaître, une option en demi-teinte. Il lui fallait pouvoir à la fois : interpréter les tendances globales et particulières à l'échelon national des crimes et délits saisis par la police et la gendarmerie; prendre ses distances avec le contenu de la communication politique de l'action du ministre lui-même ; et pratiquer une autre pédagogie à l'usage des médias en expliquant le progrès constitué par la différenciation des agrégats ventilés par modalités proactives ou réactives de leur construction. Et, à l'encontre d'une rhétorique extérieure très répandue sur la prétendue «falsification » des chiffres à la base du recueil et de toutes les étapes de leur homogénéisation, il fallut à l'OND ainsi justifier d'un usage statistique possible des séries existantes, quitte à pratiquer un contrôle post hoc de leur cohérence : "Il existe aujourd'hui bien trop de "régularités" statistiques dans les divers domaines étudiés pour faire sérieusement croire à une incidence généralisée des falsifications à la base sur le produit des résultats globaux. Il faudrait pour pouvoir en accréditer l'hypothèse que des ententes sur les fraudes à pratiquer le soient dans toutes les unités, à tous les échelons, dans une temporalité et sur des contentieux identiques au sein de toutes les polices pour que leur incidence devienne consistante et leur donne un crédit autre que purement "marginal” 37. " Ce qui n'empêcha nullement la survenue d'une épreuve de vérité ultérieure lors de la parution d'un ouvrage fort bien argumenté 38 , instruisant une thèse polémique sur le retour en force de la falsification des chiffres de collecte pour cause de "pression aux résultats " parmi les agents de première ligne, "sous l'ère Sarkozy" notamment ${ }^{39}$. On vit alors la

36. Laurent MUCCHIELLI, «Le nouveau management public à l'épreuve : délinquance et activité policière sous le ministère Sarkozy, 2002-2007 », Champ pénal/Penal Field, V, 2008, <http://champpenal.revues.org/3663>.

37. Frédéric OCQUeTEAU, «Point de vue. De la fiabilité de l'enregistrement des crimes et délits », in OND, Rapport 2005, p. XLIII.

38. Jean-Hugues Matelly et Christian Mouhanna, Police, des chiffres et des doutes. Regard critique sur les statistiques de la délinquance, Paris : Michalon, 2007.

39. Pour une vue alternative de l'enjeu des effets de la "pression politique aux résultats » dans les services de police, et notamment dans le domaine des contentieux routiers, voir Anaïck PURENNE et Jérôme AUST, 
DGGN s'efforcer d'interdire la publicité d'un tel débat ${ }^{40}$, opération de censure que le conseil d'orientation repoussa. La DGGN fut plutôt invitée par l'OND à faire un point public sur ses méthodes de recueil, à partir de sa propre base, dite JUDEX, et surtout à rendre compte des aberrations détectées lors de la mise en correspondance des affaires signalées et élucidées par ses soins. Cette épreuve de vérité interne constitua un utile défi pour un conseil d'orientation cherchant à ne pas se laisser dicter sa conduite par des directions centrales encore assez peu habituées à se justifier de leurs contradictions entre dire et faire. L'Observatoire y gagna certainement dans le renforcement de sa crédibilité à l'égard des parties prenantes. Ajoutons qu'un facteur structurel de poids favorisa le sens de l'issue de ce rapport de forces. En effet, la nécessité politique pressante d'harmoniser les indicateurs de " résultats » de la police et de la gendarmerie en matière de sécurité fit son apparition en 2003 sous le coup des contraintes de la nouvelle loi organique des lois de finances (LOLF) du $1^{\text {er }}$ août 2001 censée entrer en application en janvier 2007. Rappelons que le fondement de cette loi consiste, pour la représentation parlementaire, à pouvoir juger des "performances" annuelles des administrations, en vue d'être éclairée sur la nécessité d'en reconduire les budgets en meilleure connaissance de cause. Le Sénat fut la première assemblée à juger du travail entrepris par les deux administrations centrales, en vertu des articles 51 et 52 de la loi. Le rapport Arthuis 41 fit apparaître explicitement les raisons des hésitations des directeurs respectifs de la Police et de la Gendarmerie, dues à un trait culturel professionnel enraciné au sujet des implicites de la conception de l'efficacité à la tête des deux directions. À l'occasion d'une question anodine portant sur l'évaluation des taux de dépistage d'alcoolémie, en effet, la teneur des divergences d'appréciation fut particulièrement mise en lumière. Pour le directeur de la DGGN à cette époque, «la différence existant entre les indicateurs relatifs aux taux de dépistage d'alcoolémie [résulte] d'une divergence de conception, puisque l'augmentation de ce taux pour la police [correspond] à une intensification de leur travail, alors qu'une baisse de ce taux [signifie] pour la gendarmerie l'atteinte de l'objectif, à savoir une diminution des personnes prenant le volant après avoir consommé de l'alcool »42. L'OND s'avisa que, pour parvenir à une compatibilité réelle des nomenclatures de la police et de la gendarmerie à partir du fichier STIC-FCE (de la police) et du fichier JUDEX (son homologue de la gendarmerie), celle-ci ne deviendrait possible qu'à deux conditions : d'une part, que fussent communément assimilées les explications pour lesquelles le rapport de ces institutions aux parquets divergeait et, de l'autre, que fussent trouvées, à trois, des solutions de compromis laissant à chaque administration le loisir de fonctionner comme elles l'avaient toujours fait. La pédagogie pratiquée devait surtout viser à atténuer la tension au sujet des catégories d'infractions contenues au sein de «l'agrégat des IRAS », et résoudre par des moyens juridiques

"Piloter la police par les indicateurs? Effets et limites des instruments de mesure des performances", op. cit.

40. OND, Rapport 2007, p. 259-280.

41. Journal Officiel, 2 mars 2005.

42. Audition du Général Parayre, Sénat, Commission des finances, J.O., 2 mars 2005. 
appropriés les raisons pour lesquelles s'observaient des distorsions considérables dans les taux d'élucidation respectifs par rapport aux faits enregistrés. La mise en cause des données de la Gendarmerie par des observateurs extérieurs, Jean-Hugues Matelly et Christian Mouhanna, invités à défendre leur thèse devant le conseil d'orientation de l'OND, aida à dépasser cette question ultra sensible. Ils expliquèrent, lors d'une séance tendue, les raisons des distorsions considérables rencontrées de part et d'autre dans «l'agrégat des IRAS ", montrant comment la Gendarmerie nationale, à partir de son propre fichier JUDEX, enregistrait des taux aberrants de résolution d'affaires signalées qui n'auraient jamais dû dépasser les $100 \%$, comme c'était le cas dans la Police nationale. Les solutions trouvées à l'issue de cette épreuve démontrèrent que les mécanismes organisationnels et bureaucratiques de la saisie et de l'élucidation des affaires restaient largement gouvernés par la défense de savoir-faire très différemment institués avec le temps, ce qui n'empêchait pas les deux administrations de réagir de la même manière, quoique avec des intensités variables, aux représentations communes qu'elles partageaient des tendances de la criminalité générale annuelle. L'épreuve mit en lumière deux phénomènes jusqu'alors mal saisis des spécialistes des statistiques policières : quand les impulsions politiques confortent des réflexes organisationnels basés sur la défense de la loi et de l’ordre - le prétendu « cœur de métier », autrement dit les activités de police judiciaire -, les statistiques des crimes et délits semblent mieux maîtrisées du point de vue des performances des deux administrations. À l'inverse, quand les hommes politiques cherchent à ouvrir plus largement les polices à la plainte des victimes aux guichets, comme cela avait été notamment le cas lors de la mise en place de la réforme dite de la «police de proximité » de 1998 à 2002, les distorsions signalements/élucidations augmentaient dans les enregistrements statistiques respectifs des deux administrations. Et ce fut sans doute là l'une des raisons politiques non avouées qui présidèrent à la volonté politique de mettre fin autoritairement à l'expérience de cette réforme ${ }^{43}$.

\section{La cellule logistique, gare de filtrage et de triage et vecteur d'innovations}

La logistique de la petite équipe des neuf techniciens et chargés d'étude dirigée par un « criminologue » assisté d'un statisticien détaché de l'INSEE prit rapidement le pas sur le conseil d'orientation de l'organisme lui-même. Les agents de cette cellule furent en effet rapidement chargés de préparer les ordres du jour mensuels où étaient actées les décisions du conseil d'orientation, et de rendre compte régulièrement de leur exécution. Sa productivité est attestée par le nombre croissant de ses publications sur des supports grand public et par la diffusion rituelle d'un rapport annuel rassemblant toutes les informations recueillies au cours de l'année, moment crucial de sa propre stratégie de communication publique. Trois innovations liées à son investissement méritent examen: les bénéfices engrangés par la publication

43. Sur d'autres raisons plus structurelles de l'échec de cette réforme, voir Sebastian RochÉ, Police de proximité. Nos politiques de sécurité, Paris : Seuil, 2005. Sur les circonstances de l'annonce politique de son enterrement, voir Jean-Pierre HAVRIN, Il a détruit la police de proximité, Paris : Jean-Claude Gawsewitch, 2010 . 
mensuelle des statistiques; la stratégie de promotion d'une enquête nationale de victimation construite avec l'INSEE; et la valorisation d'autres sources policières encore sous-exploitées, issues notamment de mains courantes informatisées.

\section{La publication mensuelle des statistiques}

La décision politique, antérieure à la naissance formelle de l'OND, de publier mensuellement sur un site en ligne les données de «l'état 4001 » des crimes et délits afin de normaliser les pratiques en vigueur dans d'autres nations moins frileuses à ce sujet provoqua de fait un bouleversement dans les méthodes de communication traditionnelles antérieures. La collecte mensuelle et l'analyse des données policières et gendarmiques augmentaient considérablement le travail de pédagogie interne et de communication externe. Pour pouvoir en outre comparer mois après mois l'évolution des différents "agrégats " mis au point par l'OND, les services de collecte furent incités à arrêter l'enregistrement des faits portés à leur connaissance le 6 de chaque mois suivant. La présentation mensuelle s'accompagna d'un mode de lecture original, une analyse de l'évolution des statistiques en " mois glissants " apportant une lecture différente de ce qu'avait permis durant vingt-cinq ans la seule comparaison des données générales d'une année sur l'autre, avec les deux rectifications semestrielles dites de "consolidation ». L'opération fut rendue possible par le secours d'un argument facilitateur : l'atténuation des phénomènes saisonniers affectant des temps courts. Cette nouvelle procédure ${ }^{44}$ permit de dédramatiser des événements conjoncturels qui avaient tendance à faire monter les statistiques de crimes et délits de tel agrégat, visualisées sur une pente baissière bien plus longue.

La capacité d'innovation symbolique et la stratégie de communication systématique envers les médias de la cellule s'expliquent largement par sa position de proximité et de dépendance avec la direction de l'INHES, qui lui assure une grande facilité de négociation avec les directions centrales, membres administratifs les plus puissants du conseil d'orientation. Cette petite cellule logistique s'apparente à l'image d'une gare de triage appelée à ventiler les demandes admissibles par rapport aux faisables, et surtout à repousser «l'impossible ", après qu'eurent été négociées dans la coulisse les raisons politiques des résistances et des blocages éventuels avec les directions centrales.

Un pointage de sa productivité effectué à l'automne 2009 atteste de la publication quadrimestrielle de 20 numéros de sa revue Grand Angle, vulgarisant le fruit d'études internes thématisées, validées à partir du traitement des sources statistiques recueillies. Ces études se bornent généralement à des commentaires statistiques plus descriptifs qu'analytiques, les traitements effectués n'allant guère plus loin que la présentation sommaire de tris croisés. Si elles abordent souvent des questions originales et inédites, elles livrent rarement des analyses multivariées approfondies, faute de financements et peut-être de compétences internes, mais plus vraisemblablement encore faute d'une réelle autonomie d'action dans la véri-

44. La disjonction des commentaires de l'OND avec les commentaires «politiques» des services du ministre de l'Intérieur fut actée en janvier 2006, quand l'OND fut autorisé à diffuser ses propres analyses dans des bulletins séparés. 
fication d'hypothèses solides, capables de contredire ou d'invalider la pertinence des messages politiques affichés ${ }^{45}$. C’est évidemment sur ce point que réside la limite principale du travail de la cellule opérationnelle de l'Observatoire. Mais, pour autant que son souci des considérations méthodologiques et de transparence des données ne puisse être pris en défaut à partir des annexes qui suivent ses publications, chacun devrait pouvoir en principe s'emparer des données publiques et conduire, en toute indépendance, des analyses critiques plus approfondies 46 .

S'agissant des activités de triage de la cellule, d'autres dimensions demandent à être soulignées. Par exemple, il s'est trouvé que nombre de demandes d'études des élus au sujet de l'impact des polices municipales sur les évolutions de la délinquance se sont heurtées à des fins de non-recevoir, de même qu'ont régulièrement échoué des demandes d'aide à la création d'observatoires locaux de délinquance (OLD). Au total, quand une demande issue d'une direction centrale ou de différentes administrations paraît au conseil d'orientation ressortir de son mandat, bien que la cellule d'expertise hésite à la prendre en charge faute de moyens, une solution dilatoire se négocie en général de la sorte : la réponse immédiate est de créer un sous-groupe de travail ad hoc ayant vocation à analyser la nature des obstacles, les moyens de les circonvenir, et de proposer le faisable. On peut noter à ce sujet que quelques demandes pressantes pour construire un nouvel outil d'anticipation des « violences urbaines » ont été également repoussées par le conseil d'orientation relayant la cellule. Des débats internes ayant été vifs autour de la consistance de cette notion, l'OND acheva de se convaincre en effet de rejeter la pertinence de l'indicateur national des violences urbaines existant (INVU) pour deux raisons : au regard de l'échec de ses avatars historiques successifs, et à la faveur surtout de la cuisante déconvenue suscitée par sa faible prédictivité au regard de l'ampleur de la «crise des banlieues" de l'automne 2005 47. De même le conseil est-il parvenu à faire abandonner, contre l'avis du président de la République et de la DGPN hostile à cette offensive, un ancien indicateur en usage dans les services de sécurité publique, dit de «délinquance de voie publique » (DVP), au prétexte de son impureté : il mêlait entre autres des actes de violence commis sur la voie publique et des cambriolages chez des particuliers. Cet outil ne disparut pas complètement au sein de la DGPN qui tenait à continuer à s'en servir, donc à l'alimenter elle-même pour

45. Jean-Hugues Matelly et Christian MouhanNA, «L'OND : réussite politique et limites scientifiques indépassables? ", in OND, Rapport 2008, p. 623-634.

46. Ce que font d'aucuns, en se basant sur la publication de la direction centrale de la Police judiciaire (DCPJ) qui n'a jamais renoncé à diffuser, depuis 1972, son propre «état 4001 " annuel, même si elle le fait généralement avec beaucoup de retard, en présentant invariablement la statistique des faits constatés par les services de police et de gendarmerie (hors infractions routières) à partir d'un tableau, l'état 4001, de 107 lignes (intitulés d'infractions ou de groupes d'infractions) et de treize colonnes (faits constatés, faits élucidés, gardes à vue de 24 heures maximum, gardes à vue de plus de 24 heures, personnes mises en cause, laissées en liberté, écrouées, français, étrangers, hommes mineurs [18 ans], hommes majeurs, femmes mineures, femmes majeures).

47. Olivier HASSID, «Les violences urbaines de l'automne 2005. Autopsie d'un phénomène inédit», in OND, Rapport 2007, p. 145-155 ; Frédéric OCQUETEAU, « Les émeutes urbaines de l'automne 2005 : cadres d'analyse et points aveugles de la sociologie française», Sociologie du travail, 49 (4), 2007, p. 531-543; Hugues LAGRANGE, "The French Riots and Urban Segregation", in David WADDINGTON, Fabien JobARD et Mike KING (eds.), Rioting in the UKand France: A Comparative Analysis, Cullompton : Willan, 2009, p. 107-123. 
justifier du déploiement urbain des brigades anti-criminalité. Il fut alors symboliquement et cosmétiquement troqué par un indicateur dit de "délinquance de proximité " (DDP), parce que l'inclusion des cambriolages qu'il comptabilisait depuis des lustres parut soudain extravagant au regard de la notion véhiculée par le sigle lui-même (« voie publique»).

\section{Une enquête nationale de victimation périodique}

Mais c'est la décision de construire tous les deux ans une enquête nationale de victimation 48 , relayant et complétant une enquête plus ancienne sur les conditions de vie des ménages conduite par l'INSEE, qui devint rapidement le plus important argument de vente du savoir-faire propre à la cellule logistique de l'OND 49 . La reconstitution critique de l'histoire de ce rapprochement par des sociologues extérieurs à l'organisme en a été retracée de manière quelque peu biaisée ${ }^{50}$, s'agissant du reproche qui lui fut adressé d'avoir fragilisé des dispositifs de connaissance antérieurs solidement établis. L'INSEE, présent au conseil d'orientation de l'OND et de plus en plus étroitement associé par l'entremise de l'un de ses agents ${ }^{51}$ devenu la véritable cheville ouvrière de la cellule et la caution scientifique de sa légitimité ${ }^{52}$, aurait prétendument pris le risque de casser une série routinisée depuis 1995. Car cette série, pensée à l'époque dans le cadre d'une politique de lutte contre les exclusions sociales des ménages, aurait été abandonnée, en 2007, par la nécessité de construire, comme l'affirment ses contempteurs, une nouvelle série plus en phase avec les préoccupations et demandes de sécurité des Français. Il vaut la peine de s'appesantir sur cet argument. En effet, la décision de promouvoir une enquête nationale de victimation annuelle co-conduite par l'OND et l'INSEE, capable d'apporter une information complémentaire voire alternative aux statistiques administratives et de mettre la France au niveau des standards des nations industrielles développées, constitua l'argument le plus décisif pour obtenir des fonds de la tutelle, avant de devenir réalité. Des exemples étrangers servirent de modèle, tel le British Crime Survey, dont les instigateurs avaient incité depuis quinze ans les différents pays de l'Union à les rejoindre aux fins de comparer et de hiérarchiser, dans une démarche de benchmarking, l'intensité des demandes de sécurité adres-

48. Sur l'ancêtre de ce type d'enquête, voir Jean-Paul GRÉMY, «Première enquête nationale de victimation : le National Crime Survey", in OND, Rapport 2007, p. 103-110.

49. Voir le « dossier» récapitulatif, in OND, Rapport 2008, p. 37-194.

50. Emmanuel Didier, Sophie NÉVANEn, Philippe RoBert et Renée ZAUBERMAN, « La solidité des institutions. Les statistiques de victimation de l'INSEE 1996-2006", Genèses, 74 (1), 2009, p. 128-144. Voir aussi, plus technique et moins polémique, Lise Miceli, Sophie NÉvanen, Philippe RoberT et Renée ZAUberman, «De l'instantané au long métrage. L'enquête Cadre de vie et Sécurité dans la série des données sur la victimation ", Économie et Statistiques, 426, 2009, p. 1-28. Très curieusement, au sujet des travaux de l'OND, on lit ceci, dans la note 6 de la page 7 de cet article : «De son côté, une agence de ministère de l'Intérieur a fait figurer dans ses rapports annuels (sic; la référence signalée dans le texte concerne le premier rapport de 2005) quelques exercices partiels de mise en série portant sur les enquêtes de l'INSEE pour les années 1996, ou 1998 selon les cas, à 2003. "

51. Attaché principal, cet agent a été mis à disposition en 2003. Par suite du changement du statut de la tutelle (INHES puis INHESJ), il est détaché dans l'établissement public depuis 2007.

52. Stéphan LOLLIVIER, «L'utilisation des statistiques à l'OND. Un bilan flatteur mais des pistes de progrès encore nombreuses ", in OND, Rapport 2008, p. 635-645. 
sées par les victimes aux autorités de chacun des pays adhérant à cette enquête internationale ${ }^{53}$. Les résultats avaient semblé prometteurs, mais il faut bien reconnaître que ni le pilotage étranger ni les investissements intellectuels et financiers français ne furent jamais à la hauteur de ce qu'il devenait urgent pour l'État de construire sur son propre territoire. Trois ans de négociations furent donc nécessaires pour finaliser la réalisation d'une enquête nationale "sous pilotage de l'Intérieur » (2007), trouver les fonds pour la co-construire avec l'INSEE, expérimenter des dispositifs transitoires (2005 et 2006) afin surtout de la rendre d'une lecture compatible avec les sources policières disponibles. Un objectif dont ne s'étaient jamais directement souciés, avant $2004^{54}$, les sociologues qui avaient importé et expérimenté ce type d'enquête en France dans les années 1980. Il fallut, vingt ans plus tard, à l'OND rassurer tant l'INSEE que le ministère de l'Intérieur sur les objectifs réels d'une telle comparaison, et vaincre de nombreuses inhibitions liées aux anticipations de ses usages politiques et sociaux perçus comme allant échapper aux administrations nationales, au point de remettre en cause leurs certitudes. Craintes et préjugés restaient tenaces à ce sujet, car les différentiels d'information sur la quantification des crimes vus par les polices (évolution des crimes et délits issus de "l'index 4001 ») et par les victimes 55 sondées sur le vécu réel de leur insécurité (réelle ou imaginée) avaient jusqu'alors provoqué sur tel ou tel contentieux des explications antithétiques au sujet de la nature de la confiance ou de la défiance des citoyens envers les agences policières.

Il faut bien reconnaître que, du côté de la sociologie française, les positions scientifiques étaient longtemps restées tout aussi figées sur le bien-fondé de ce type de confrontation de sources, bien qu'elle ait commencé à les admettre sous la décennie 2000. Mais, puisqu'à l'étranger, les enquêtes nationales et locales auprès des victimes passent pour des outils de connaissance tout à fait légitimes afin de mieux

53. Voir Frédéric OCQUeTEAU, Jacques FrenAIs et Pierre VARLY, Ordonner le désordre, Paris : La Documentation française, 2002, p. 106-108, à propos des premières tentatives de comparaisons de l'enquête internationale ICVS (International Crime Victims Survey). Sur l'analyse des résultats comparés, voir : Pat MAYHEW et Jan VAN DIJK, Criminal Victimization in Eleven Industrial Countries. Key Findings from 1996 International Crime Victim Survey, La Haye: WODC, 1997, pour la réplication de l'enquête 1996 menée dans 11 pays; John VAN KeSTEREN, Pat MAYHEW et Paul NieuwbeERTA, "Criminal Victimization in Seventeen Industrial Countries: Key Findings form the 2000 International Crime Victim Survey ", La Haye : WODC-NSCR, 2001, pour l'enquête 2000 menée dans 17 pays ; Michael R. RAND, «The National Crime Victimization Survey at 34: Looking Back and Looking Ahead ", in Michael MAXFIELD et Mike Houg (eds.), Surveying Crime in the 21st Century, Cullompton: Willan, 2007, p. 145-164, pour l'enquête conduite en 2004 dans 34 pays. Précisons que sur les quatre vagues de l'enquête ICVS, 1989, 1992, 1996 et 2000, Angleterre et Galles, Canada, Finlande, Hollande et USA financèrent leur National ICVS à quatre reprises, tandis que la France le fit à trois reprises (sur un financement IHESI), comme l'Australie, la Belgique, le Japon, l'Irlande du Nord, la Pologne, l'Écosse, la Suède et la Suisse. On ne peut donc pas vraiment soutenir que les pionniers français aient inventé la méthode, tout au plus ont-ils eu l'immense mérite de l'y avoir acclimatée. Appartenant pour leur propre logistique aux services du ministère de la Justice, on doit surtout leur reconnaître le mérite d'avoir permis à d'autres "agences " de continuer dans cette voie de l'acclimatation nationale d'une enquête routinisée par le biais de financements administratifs alternatifs (ministère de l'Intérieur, puis services du Premier ministre).

54. Hugues Lagrange, Marie-Lys Pottier, Renée Zauberman et Philippe Robert, « Enquêtes de victimation et statistiques de police : les difficultés d'une comparaison ", Déviance et société, 28 (3), 2004, p. 285-316.

55. Renée ZAuberman et Philippe Robert, Du côté des victimes, un autre regard sur la délinquance, Paris : L'Harmattan, 1995. 
guider l'action policière dans des contextes mal outillés par ailleurs, les différents arguments finirent par avoir raison des résistances administratives. C'est ainsi que la cellule statistique de l'OND, au prix surtout d'un effort de rigueur dans la méthode et d'une pédagogie de réassurance constante auprès des directions centrales (qui avaient toujours le sentiment de pouvoir contrôler son travail), parvint enfin à comparer, en 2007, les résultats des principales sources de connaissances des crimes et délits, commis et subis, à l'échelon national. Elle parvint à réitérer l'opération deux ans plus tard, sans drame majeur, démontrant que cet exercice était possible et souhaitable, à la fois « hors et sous » contrôle de la police et de la gendarmerie.

\section{La valorisation d'autres sources policières}

La valorisation de données nationales au sujet des mains courantes informatisées fut la troisième des innovations initiées par la cellule. Elles mirent plus du temps à être présentées comme une autre source policière précieuse à la connaissance des évolutions de la sûreté, comme si elles n'étaient qu'une source mineure de connaissances, et par suite pouvait être négligée dans le débat public. Or, il apparut que cet outil traditionnel de gestion des ressources opérationnelles sur le terrain pouvait tout aussi bien, sinon mieux, renseigner sur l'étendue du vaste champ de la conflictualité sociale liée aux infractions contraventionnelles, ou du domaine infra pénal des incivilités (ou petits désordres) perçus par la police. Cette source de connaissances à standardiser dans un but de quantification sous des catégories de faits pertinentes dut néanmoins subir le test de sa rationalisation technique en vue de démontrer ses différentes utilités pratiques. C’est dans la montée de l'idéologie de la "satisfaction du public » aux guichets des commissariats que cet instrument de quantification prit alors une nouvelle légitimité. Il permettait en effet non seulement aux pouvoirs publics de mieux appréhender la nature des « attentes du public » par rapport aux dépôt de plaintes des victimes dans les commissariats, mais plus encore d'offrir une plus juste appréhension du destin de ces plaintes par types de "contentieux" problématiques. La rationalisation informatique de la main courante, pensée au milieu des années 1990 et généralisée dix ans plus tard sur l'ensemble du territoire, autorisait la poursuite de deux objectifs : mieux justifier des actions policières entreprises dans le domaine contraventionnel 56, mais aussi et surtout mieux justifier les réponses apportées aux élus sans vue d'ensemble sur l'intensité relative des phénomènes d'insécurité liés aux actes incivils ou aux perturbations de l'ordre urbain local. On savait que des commissaires de la sécurité publique se servaient abondamment du contenu de cette source pour augmenter leur propre pouvoir et leurs marges de manœuvre dans les réseaux de partenariats au sein de certaines municipalités sensibles, au sein de dispositifs tels que les CLSPD, par exemple 57 . De nombreuses monographies avaient assez correctement identifié comment certains commissaires aidaient des édiles à mieux com-

56. Les contraventions de toutes catégories notamment. Voir OND, Rapport 2008, p. 287-302 ; Jean-Luc BESSON, "Les signalements d'usagers enregistrés dans la main courante informatisée de la préfecture de police de Paris en 2008 ", in OND, Rapport 2009, p. 231-246.

57. Tanguy LE GoFf, Les maires, nouveaux patrons de la sécurité. Étude sur la réactivation d'un rôle politique, Rennes : Presses universitaires de Rennes, 2008. 
prendre les phénomènes d'insécurité sur leur propre territoire ${ }^{58}$. Or, les gisements d'information induits à propos des pathologies urbaines, incivilités et autres nuisances environnementales mis en évidence par cette source enregistrant le désordre signalé par le tout venant, auraient pu aider maires et services techniques à mieux réagir aux préoccupations de leurs concitoyens, quand l'espoir naquit de pouvoir construire à ce sujet des observatoires locaux de la délinquance (OLD). Mais les nombreuses tentatives ayant cherché à les mettre en place, dès la naissance de l'OND, tournèrent court. La crainte restait trop forte, parmi la plupart des commissaires de police, de se voir alors « dépossédés » de leur seul outil de pilotage interne, s'ils avaient dû le partager avec des maires et leurs polices municipales parfois perçus comme des concurrents illégitimes et mieux dotés que la Police nationale elle-même. C'est pourquoi la main courante informatisée (MCI) 59 demeura longtemps un outil d'action sous-investi, les directions centrales préférant le présenter comme un outil exclusif de management des fonctionnaires sur le territoire national. En réalité, la MCI permet d'accroître considérablement la connaissance interne du travail d'une police exerçant dans son tissu environnemental, rendant possible un enrichissement de l'information sur le « rendement » effectif de la sécurité publique dans le domaine de la sécurisation urbaine ${ }^{60}$. Elle permet en effet d'y tester, en temps réel, l'intensité de la conflictualité relative par les taux d'occupation de l'espace et l'investissement dans la diversité des tâches de chaque policier ou équipe de police. Qu'il s'agisse d'îlotage, de traque, d'interpellation, de recueil de plainte, ces différentes tâches plus ou moins autonomes sont censées être reliées aux signalements des crimes, délits, contraventions, accidents, différends, nuisances signalés de toutes parts. Une meilleure transparence y est de mise, puisque les agendas des horaires individuels des interventions effectives et actions entreprises peuvent être suivis en temps quasi réel. Les chefs de service et officiers pilotent mieux l'exécution ou l'inexécution des consignes dans les divers quotas d'opérations administratives et judiciaires, partenariats et actions de communications demandés. On comprend aisément l'utilité de cet outil, mais également pour-

58. Par exemple, Philippe PALOMO, "Observatoire de la sécurité et analyse spatiale de la délinquance: quelle démarche méthodologique ?», Paris, Mémoire pour le DESS Ingénierie de la sécurité, IHESI/Paris V, 2000 .

59. La MCI est devenue un outil de pilotage en temps réel des forces policières sur les points chauds de l'insécurité. L'exploitation des informations saisies vise, d'une part, le suivi chronologique des événements (174 codes) et leur mode de saisine sur l'ensemble du territoire, d'autre part, les déclarations et signalements des usagers pour des faits n'entrant pas dans la nomenclature de l'état 4001. L'effet principal de la généralisation de cet outil a été de dynamiser les effectifs à travers le " test d'emploi des personnels ", une technique mise au point par l'IGPN pour évaluer les modalités d'occupation policière de la voie publique, par le croisement des taux de présence des effectifs dans la rue, et donnant la possibilité de vérifier la cohérence entre la distribution horaire et journalière des effectifs et la répartition temporelle des phénomènes d'insécurité. Voir Jacques RotivaL, "La main courante informatisée, une clé stratégique de la modernisation de la Police nationale», Les Cahiers de la sécurité, 61, 2006, p. 203-214. Pour une première lecture nationale de l'apport des déclarations en MCI, voir Rachid BÉNAZRINE, «Zoom sur les déclarations de mains courantes enregistrées dans les départements métropolitains et ultramarins en 2008 ", in OND, Rapport 2009, p. 400-406.

60. Cf. Frédéric OCQUeTEAU, La sécurité publique à l'épreuve de la LOLF, op. cit.; ID., "Qu'est-ce qu'un service de sécurité publique rentable?», op. cit. 
quoi il suscite autant de réticences parmi les agents de première ligne et leurs syndicats qui y voient un inadmissible moyen de contrôle de leur traditionnelle autonomie d'action au quotidien ${ }^{61}$. En discuter publiquement des finalités pratiques (contrôler la population ou contrôler le travail policier) s'est donc avéré un enjeu éminemment problématique, en dépit de sa seule raison d'être officielle : doter l'État d'une meilleure connaissance évaluative de l'implication personnelle des fonctionnaires de la sécurité publique, depuis l'entrée en vigueur de la LOLF et de l'entrée officielle dans l'ère de la RGPP. Pris dans l'étau de ces deux attitudes paralysantes, force est de reconnaître aujourd'hui que cet outil n'a pas permis d'accompagner une réflexion collective sur les moyens de restaurer de la tranquillité sur des espaces troublés, autrement dit de discuter très précisément de l'imbrication des finalités de la prévention, de la dissuasion ou de la tolérance policière, parce que ses usages méconnus restent encore trop limités, pour ne pas dire verrouillés ou tabous.

\section{Conséquences de la montée en légitimité politique de l'Observatoire}

Le péché originel du conseil d'orientation de l'Observatoire sous examen provient des circonstances de sa naissance étroitement encadrée par la DGPN, courroie de transmission et porte-parole jalouse des seuls intérêts "sécuritaires " défendus par ministère de l'Intérieur. Ce relatif étouffement institutionnel obligea néanmoins l'organisme à exister dans une sorte de double bind durant quelques années, avant de conquérir son propre espace de légitimité. Il réussit partiellement à s'émanciper de sa tutelle native en se donnant des objectifs de connaissance alternatifs (enquêtes de victimes), en rationalisant les données existantes dans une visée de mise en commun pour aider des administrations proches et rivales qui n'y seraient jamais parvenues à elles seules. Pour autant, l'OND ne parvint jamais à mobiliser significativement à sa cause fédératrice l'ensemble des administrations, et notamment, les plus difficiles d'entre elles, les services de la Chancellerie, ce qui fit longtemps douter de sa crédibilité. C'est pourquoi, le jour où fut levée l'hypothèque que la «créature de l'Intérieur » pourrait échapper à son contrôle parce que des techniciens des statistiques judiciaires et pénitentiaires y trouveraient leur place, cette malédiction originaire put enfin être levée. Et comme c'est très souvent le cas en France, cela ne put passer, symboliquement, que par un changement institutionnel à l'égard de la nouvelle tutelle de rattachement, autrement dit que le Premier ministre devienne l'autorité d'arbitrage éventuelle entre les rivaux, en créant une nouvelle structure rattachée à son service où seraient mieux rééquilibrés les intérêts de connaissance du crime par les services de la Chancellerie et ceux de l'Intérieur. Techniquement, le processus de rapprochement institutionnel ne pouvait véritablement se concrétiser qu'à partir du moment où la question de la traçabilité des «mis en cause» permettait d'aplanir les rapports de rivalités empoisonnés entre les deux institutions. En voici les étapes significatives.

61. Sur les conséquences de ce mécanisme en termes "d'involution des buts ", voir l'analyse classique de Dominique MONJARDET, Ce que fait la police, sociologie de la force publique, Paris : La Découverte, 1996. 
Dès 2005, un diagnostic assez sévère est établi au sujet de l'absence de continuité entre les statistiques du ministère de l'Intérieur et celles du ministère de la Justice à partir des dénombrements des «mis en cause » et des " affaires" 62 . La source de «l'état 4001 » et les statistiques recueillies par les cadres du parquet sont depuis longtemps largement incompatibles, mais jusqu'alors, personne n'avait pu, voulu ou su porter remède aux chevauchements et aux distorsions des procédures. Le diagnostic établi est à peu près constant : $70 \%$ des procédures policières et gendarmiques établies par PV arrivant à la connaissance des parquets passent à la trappe, faute d'enregistrement. La cause en est principalement imputée au fait que les PV reçus n'entrent généralement pas dans les schémas de la procédure judiciaire utile. Les données issues des nomenclatures utilisées par les agences policières pour qualifier les faits sont certes enregistrées par les bureaux d'ordre selon une nomenclature correspondant à des faits signalés à la Justice (NATAFF), mais ces affaires, pénalement requalifiées par les parquets sous une autre nomenclature (NATINF), obéissent à une modalité d'enregistrement exclusivement juridique, calquée sur des incriminations telles qu'écrites dans le Code pénal. Il en résulte le constat d'une énorme déperdition d'informations quantifiées, ce que le politique, relayant une interprétation spontanée des médias, a tendance à interpréter comme une mauvaise volonté de coopération de l'administration judiciaire, pour ne pas évoquer une prétendue attitude laxiste des juges. Le passage des «infractions constatées » aux unités de mesure liées à une " affaire " ou à une "personne " rend à peu près illisible la cohérence de la chaîne pénale, et malaisée toute spéculation sur l'impact des mesures prises en cas de récidive légale ou formelle d'un auteur. Dès 2003, l'enjeu technique de la traçabilité des procédures est devenu une pomme de discorde ultra sensible entre les administrations régaliennes de l'État. Car assurer la traçabilité des procédures croisées avec le nom d'une personne (policièrement suspectée mais judiciairement innocente jusqu'à l'établissement de sa culpabilité pénale) signifie pour tous les acteurs l'acceptation de se doter des moyens techniques capables de mettre en rapport criminalité constatée et criminalité sanctionnée, à partir d'un nœud de lisibilité des différentes réponses apportées par la trace de l'action des parquets.

Un groupe de travail, mis en place en 2004 sur cette question, est jusqu'alors resté sans effets décisifs, en dehors de la solution d'accélérer les transmissions télématiques entre ministères. L'OND s'empare de l'enjeu et revient annuellement à la charge, obligeant, avec les formes requises, la Chancellerie à réactiver un groupe de travail capable de se donner les moyens intellectuels et techniques de mettre au point un "numéro unique» de suivi sécurisé pour toutes les procédures entrant dans le circuit policier, et surtout d'en donner à voir les suites aux directions centrales de l'Intérieur et de la Défense, une demande lancinante apparemment jamais assouvie. La résistance de la Chancellerie à cette entreprise s'explique certes pour des raisons techniques de coûts qu'elle devrait assumer seule, sans en avoir les moyens, mais également parce qu'il se joue là un enjeu symbolique extrê-

62. OND, Rapport 2005, p. 546 et suiv. 
mement inhibiteur : celui du sentiment d'une forme de dépossession de l'autorité judiciaire par l'administration policière. À l'époque, l'OND est encore largement perçu comme une "créature de la police " ( $\mathrm{sic}$ ), et les parquets, sous la tutelle de la Chancellerie, n'entendent pas si facilement rendre des comptes aux administrations policières au sujet de leur pouvoir d'appréciation de l'opportunité de leurs poursuites par des classements en fait et en droit, en dépit des réformes les obligeant à les justifier.

En 2006, la direction des Affaires criminelles et des Grâces 63, à nouveau mise en cause par le conseil d'orientation de l'OND à ce sujet, fait état d'un premier test expérimental sur la nouvelle chaîne pénale (NCP), mis en place au parquet de Créteil, afin de démontrer sa volonté de coopération. Un numéro unique d'enregistrement des plaintes destiné à assurer la traçabilité des procédures est testé, visant à mettre en adéquation le statut de la criminalité constatée par la police et la gendarmerie avec les réponses du parquet ${ }^{64}$. Forte d'avoir été entendue au sujet de ces contraintes techniques, la Chancellerie suggère alors que le parquet affecte un numéro « le plus tôt possible " aux PV de police primaires et secondaires, de façon à permettre l'intégration rapide d'un numéro traçant les personnes mises en cause, au sein même des bases de données des services de police et de gendarmerie. L'argument est habile qui donne aux services des parquets le soin de reprendre la main sur l'enjeu de deux façons : d'une part, en les rendant plus responsables du destin des données policières ; de l'autre, en laissant à la direction de la Sécurité publique une marge de manœuvre suffisante permettant une meilleure entente entre les deux acteurs clés de la chaîne pénale. Cette suggestion laisse surtout le loisir à la Chancellerie de renvoyer aux services de police la responsabilité de l'origine de la définition de la cohorte d'une population statistique dont il devient impératif à l'époque d'étudier le destin à l'entrée des mis en cause dans la machine jusqu'à leur sortie de la chaîne pénale (jusque et y compris leur retour éventuel en cas de "réitérations»). Cette exemplification empirique est en effet devenue un objet de controverse récurrent, s'agissant de prouver politiquement l'efficacité dissuasive de la justice pénale, au moment de la discussion sur les "peines plancher» et les projets de loi durcissant les conséquences de la récidive. À partir du moment où le numéro émetteur de l'entrée dans la machinerie pénale, ou au sein d'une cohorte, relève de la responsabilité des services de police, il revient nécessairement à ces derniers de mieux renseigner les dates du PV, d'enregistrement et de

63. OND, Rapport 2006, p. 492 et suiv.

64. Ce test porta sur 847 procédures transmises par le commissariat de Créteil. Les services de la Chancellerie expliquèrent les raisons pour lesquelles 335 d'entre elles n'avaient pu être identifiées : dans une proportion de 8 fois sur 10, parce que les auteurs n'avaient pas été identifiés par le PV, et n'avaient donc pas pu être enregistrés ; 2 fois sur 10, il s'agissait de procédures avec des auteurs connus, mais nécessitant des enquêtes complémentaires à partir du nom du mis en cause. La Chancellerie renvoya alors la balle, en se plaignant du fait que beaucoup de PV portaient un même numéro. Elle en inférait que plusieurs services de police utilisaient le même pour une affaire identique, alors qu'à ses yeux celle-ci devait faire l'objet de numéros d'enregistrement différents. Elle argumenta en outre le fait que, dès lors que plusieurs procédures étaient «jointes " par le parquet, un seul PV devait être enregistré, car seul le service ayant saisi le parquet était censé figurer dans la NCP. 
transmission des procédures initiées pour mettre fin aux errements du système antérieur.

La DGGN prit alors ses distances, arguant n'être pas concernée puisque son système de signalement propre rendait impossible la moindre marge d'autonomie dans l'attribution d'un numéro de PV à deux procédures différentes. Durant cette nouvelle épreuve de force bureaucratique où des réputations et des savoir-faire étaient à défendre, elle trouvait sa revanche, renvoyant dos à dos non seulement les parquets et la Chancellerie à leurs propres défaillances, mais également la DGPN à ses propres responsabilités.

Dans son édition annuelle de 2007, l'OND constate que ce dossier délicat n'a pas avancé en dépit de la réactivation d'un nouveau groupe de travail ${ }^{65}$. Les modalités intellectuelles de construction du continuum ont certes été actées, mais les modalités techniques traînent, qui sont alors expliquées par des entraves liées aux applications informatiques propres à chaque ministère ${ }^{66}$. En 2008, le groupe interministériel institué entre-temps confirme avoir avancé dans la mise en œuvre de la transmission télématique des procédures pénales ${ }^{67}$. En 2009 , l'OND fait état de sa satisfaction au sujet des explications fournies par le groupe de suivi de ce comité qui affirme s'être engagé à rendre « opérables » les nouvelles applications informatiques de la Police, de la Gendarmerie et de la Justice, dès que les contraintes techniques de la mise en informatique seront surmontées. Se trouve alors confirmée la décision politique d'y importer les données issues des bulletins d'enquêtes judiciaires (de la gendarmerie) et des comptes-rendus d'enquête (de la police) ${ }^{68}$, manière de reconnaître officiellement que des pratiques officieuses de pré-qualification sont bien présentes au sein des deux administrations, contrairement à ce qu'a toujours prétendu la DGGN au sujet de l'inexistence des mémentos et « cahiers de service» personnels chez les gendarmes 69 .

En réalité, une issue à ce blocage apparemment technique a été trouvée au niveau d'acteurs d'interface jusqu'à présent négligés et restés dans l'ombre: les agents des bureaux d'ordre au sein des parquets. En effet, avec la mise en place de ce système modernisé, ils n'auront désormais plus à ressaisir les informations pour la Chancellerie puisque les nomenclatures de signalements auront été harmonisées en amont. En d'autres termes, ils n'auront plus à les «convertir » manuellement. Cette solution trouvée au "chaînon manquant" allait surtout permettre de lever fantasmes et soupçons au sujet des responsabilités des uns et des autres quant aux dysfonctionnements antérieurs.

La publicité de ces débats techniques au sein de l'OND permit surtout de comprendre qu'un acteur clé du blocage dans le séquençage de la chaîne, invisible au conseil d'orientation, était apparu comme l'acteur salvateur du déblocage, et cela

65. OND, Rapport 2007, p. 536-537.

66. Ardoise pour la DGPN, Pulsar pour la DDGN et Cassiopée pour la Justice.

67. OND, Rapport 2008, p. 742.

68. Dans l'application informatique « justice» du logiciel dit Cassiopée.

69. Sur ce point, voir François Dieu et Paul Mignon, La force publique au travail. Deux études sur les conditions de travail des policiers et des gendarmes, Paris : L’Harmattan, 1999 (spécialement, p. 200 et suiv.) 
au moment même où le politique s'apprêtait à annoncer la bonne interconnexion des fichiers de police avec ceux de l'administration judiciaire, quand la Gendarmerie allait passer sous la tutelle de l'Intérieur, à partir du $1^{\text {er }}$ janvier 2009.

\section{Conclusion}

Entre les mandats officiels de l'OND «version 2004 » et la version élargie de l'ONDRP « version 2009 » 70 , se situe un intervalle de six ans où se sont fait jour des tactiques obstinées de contournement d'obstacles liées à la résistance au changement ou à l'inertie des institutions, de tri entre l'impossible, le souhaitable et le faisable, face aux demandes d'une meilleure harmonisation d'instruments de mesure affluant de toutes parts. Les travaux de la cellule statistique de l'Observatoire, systématiquement publicisés en direction de médias cherchant à disposer de messages simples et pas nécessairement conformes à ceux du Cabinet du ministre de l'Intérieur, ont contribué à la mise en visibilité de cet OND. À la suite de quoi a pu prospérer la décision politique d'en rehausser la tutelle sous les services du Premier ministre, condition indispensable à l'enrôlement des directions de la Chancellerie dans un conseil d'orientation rénové 71 .

C'est désormais un fait acquis : l'OND se voit délivré de ses attaches natives de 2004, celles des directions centrales du ministère de l'Intérieur et de la Défense. Dans sa quête de légitimité politique, son nouveau rattachement administratif sous la tutelle des services du Premier ministre en tant qu'Observatoire national de la délinquance et des réponses pénales (ONDRP), est censé lui apporter une légitimité moins entachée de suspicion. L'opération était certes devenue nécessaire pour briser les hésitations à la coopération de la puissante direction des Affaires criminelles et des grâces du ministère de la Justice et de ses autres directions. Il n'est cependant pas acquis que le montage de ce nouveau dispositif y suffise. Il signale néanmoins une nouvelle donne : à défaut d'une reprise en main pure et simple des statistiques policières des crimes et des délits par la Chancellerie, il existe au moins pour cette administration une occasion à saisir pour renégocier sa marge de manœuvre quant à sa lecture de l'évolution quantifiée des crimes et délits suspectés et punis, en concertation beaucoup plus étroite avec les administrations policières.

On peut résumer de la façon suivante ce qu'apporte une description minutieuse à l'institutionnalisation progressive d'un dispositif tel que celui de l'OND et du rôle que joue son conseil d'orientation à propos des usages des instruments de mesure qu'il fédère, harmonise, traite et restitue au public et au politique.

Quand les outils de recueil et de traitement de données au sein d'une black box, objet de tous les fantasmes, deviennent un peu plus transparents ou moins opaques,

70. À ce sujet, il convient de mettre en parallèle la composition du conseil d'orientation tel que prévu par le décret 2004-750 du 27 juillet 2004 le plaçant sous la tutelle du ministère de l'Intérieur, et celle du conseil d'orientation de l'ONDRP tel que prévu par le décret 2009-1321 du 28 octobre 2009, placé sous la tutelle du Premier ministre.

71. Sur le rôle important joué dans cette opération par un démographe du CNRS, expert en statistiques pénitentiaires, voir Pierre-Victor TOURniER, "Vers un observatoire national de la délinquance et des réponses pénales ", in OND, Rapport 2008, p. 665-671. 
la société civile intéressée peut y gagner si les citoyens comprennent mieux à quoi et comment ils sont réellement construits et mobilisés. Les réformateurs des partis d'opposition peuvent y gagner en crédibilité s'ils apprennent à mieux ajuster leurs attaques sur la prétendue efficacité réelle des politiques, à défaut de demeurer focalisés sur les seuls ressorts de la critique en inefficience des instruments de mesure de la sûreté générale de leurs adversaires politiques.

S'agissant de la croyance en une nécessaire capitalisation de savoirs disparates pour pouvoir agir, qui anime les membres d'une structure telle que l'OND-ONDRP, autrement dit quand émerge une meilleure intelligence 72 au sujet du bricolage des instruments de mesure des insécurités, il apparaît qu'une telle conviction n'est certainement pas due qu'au fantasme intéressé du sociologue.

Quand on cherche à saisir de l'intérieur les modalités saillantes de leur mobilisation, on est obligé de les prendre au sérieux. Les outils de mesure, "acteurs pilotes » de l'action publique, sont bel et bien susceptibles de transformer les activités administratives elles-mêmes, et de modifier à la marge les finalités du métier de ses nombreux serviteurs. On le voit clairement lors de la mise à jour des jeux d'influence au sein de bureaucraties en concurrence permanente quant à la maîtrise de l'impact environnemental du travail de leurs agents de terrain, et quant aux anticipations sur les appropriations de l'efficacité performative des chiffres dans un contexte ultra politisé.

Les lectures critiques liées aux évolutions de la criminalité légale et apparente apparaissent de leur côté profondément désajustées de par l'effet de leur trop grande extériorité aux enjeux internes, constamment minimisés. Leur refus de débattre frontalement de résultats fournis par de nouveaux instruments de mesure de plus en plus banalisés avec de nouveaux acteurs dans le champ de l'expertise profane et professionnelle 73 ne laisse pas de surprendre, quand chacun sait qu'aucun instrument de connaissance ne s'impose jamais de lui-même de par sa seule autorité, puisqu'il a toujours besoin d'interprètes.

Le souci d'améliorer le stock des informations utiles vers les décideurs, les opérations de regroupement, de recherche de cohérence et d'accessibilité de données disparates pour les alerter sur de vieux problèmes remis au goût du jour, n'empêchera jamais que la connaissance collective produite par les instruments de mesure restera toujours dotée d'utilisations stratégiques en conflit parmi leurs différents interprètes. Pour autant, la multiplication des instruments de mesure affinant la connaissance de phénomènes sociaux devrait diminuer les foyers de discorde autour de leurs utilisations plutôt que de les amplifier, sauf à faire le jeu des seuls producteurs particulièrement «résistants au projet de connaître » 74 . Si l'on peut

72. Au sens de P.-A. Rosental qui, dans sa sociohistoire de la science démographique, désigne des constructions historiques simultanées entre politiques, groupes, institutions et savoirs : voir Paul-André RosENTAL, L'intelligence démographique. Sciences et politiques des populations en France 1930-1960, Paris : Odile Jacob, 2003.

73. Michel CALlon, Pierre LASCOUMES et Yannick BARTHE, Agir dans un monde incertain. Essai sur la démocratie technique, Paris : Seuil, 2001.

74. Pour reprendre la célèbre formule du regretté Jean-Paul BrodeuR : "La police, mythes et réalités ", Criminologie, 17 (1), 1984, p. 9-41. 
comprendre que, parmi toutes les administrations, les fonctionnaires de police et de gendarmerie craignent de se voir dépossédés de leurs propres données, de leur pouvoir de rétention pour conserver leur zone de maîtrise traditionnelle sur le périmètre de leur savoir/pouvoir, il serait navrant qu'un dispositif comme celui de l'ONDRP en vienne à être considéré comme participant à la consolidation de cette attitude, alors que son mandat va précisément dans le sens opposé. La vieille malédiction de Quételet est en passe d'avoir vécu, selon laquelle les techniciens du recueil des données du crime ne pourraient jamais en devenir les interprètes, à cause d'un défaut de culture légitime adéquate. Mais, face à ce préjugé bien commode, il convient d'avoir enfin le courage de poser cette question : en quoi les interprètes légitimes (prétendus plus cultivés sur le social ?) des statistiques administratives de la police en matière de crimes et délits seraient-ils plus fondés que les techniciens (prétendus moins cultivés ?) à en discuter les secrets de fabrication ? En quoi le seraient-ils, alors qu'eux-mêmes seraient bien impuissants à pouvoir fonder leur propre science si, dans le domaine de la quantification, ils ne disposaient pas d'outils de mesure sans cesse améliorés et surtout alimentés par d'autres administrations dédiées?

Il nous semble que des percées décisives ont été accomplies dans le domaine de la connaissance du travail d'exploitation des données collectées sur le crime, un work in progress permanent qui ne saurait être réduit à de pures opérations techniques d'accumulation et de stockage d'informations. Comme l'ont très bien remarqué certains analystes 75 , il importe, serait-ce dans un horizon synchrone, de mieux comprendre les « interférences complexes entre les enjeux profanes et scientifiques, organisationnels et politiques de la collecte, de la diffusion et de l'exploitation des données tant qualitatives que quantitatives ".

Tel était le pari dans un domaine particulièrement difficile d'accès et partant, sous-investigué. À notre sens, un observatoire tel que l'OND doit être lui-même soumis à une observation approfondie de l'intérieur, en ce qu'il est aussi un lieu de controverses révélatrices et fécondes, hélas encore trop souvent feutrées et confidentielles. Pour formuler l'image de ce qu'il pourrait devenir en tant qu'activité constituante consolidée dans les termes d'Alain Desrosières 76 , on pourrait sans nul doute évoquer la figure du futur ONDRP comme le lieu d'une nouvelle convention, un espace de dialogue et de débats " où les formes [seraient] perçues comme indiscutées pour que la vie puisse suivre son cours, et discutables pour faire changer le cours de la vie».

À défaut d'avoir connu un apaisement définitif des positions en ce lieu constituant privilégié, il nous semble au moins avoir levé quelques tabous sur les causes des blocages persistants dans la collecte et le rassemblement des diverses sources. Les entraves au volontarisme politique y ont été diagnostiquées, nous avons mon-

75. Michel Callon, Pierre Lascoumes et Yannick Barthe, Agir dans un monde incertain. Essai sur la démocratie technique, op. cit.

76. Alain DeSrosières, La politique des grands nombres. Histoire de la raison statistique, Paris : La Découverte, 1993. 
tré comment ont été surmontées certaines chausse-trapes et délaissées pour le moment des propositions ou entreprises réputées impossibles.

La controverse publique au sujet des « chiffres » des délinquances et des insécurités, liée à la manière dont les dirigeants politiques du moment entendent se les annexer pour mettre en scène l'image la plus flatteuse de leur action, doit être dépassée et repartir sur des bases renouvelées, y compris pour des équipes de rechange se préparant à de futures alternances politiques.

\section{口 L'auteur}

Juriste, sociologue et politiste, Frédéric Ocqueteau est directeur de recherche au CNRS, membre du Centre de Recherches Sociologiques sur le Droit et les Institutions Pénales (CESDIP), UMR 8183. Spécialiste de la sécurité privée, de l'évaluation des politiques publiques de sécurité, des professions et des identités policières, des instruments d'action policiers (vidéosurveillance, indicateurs de crime, management à la rentabilité des services), il est l'auteur de nombreux ouvrages et articles, parmi lesquels on citera :

- La sécurité privée en France (avec Daniel WARFMAN), Paris : PUF, 2011 ;

— "Chefs d'orchestre de la sûreté des entreprises à l'ère de la sécurité globale ", Champ pénal/Penal Field, VIII, 2011 ;

- «Polices et politiques de sécurité. Concilier efficacité et respect des libertés », Problèmes politiques et sociaux, 972 , mai 2010 ;

- Une mémoire policière sale: le fichier STIC (avec Philippe PICHON), Paris: JeanClaude Gawsewitch, 2010;

— Mais qui donc dirige la police? Sociologie des commissaires, Paris : A. Colin, 2006 ;

— Polices entre État et marché, Paris : Presses de Sciences Po, 2004. 